\title{
Irreversible Vasculopathy Proceeds Rapidly in POEMS Syndrome
}

\author{
Kenichi Sakuta ${ }^{1}$, Taiji Mukai ${ }^{1}$, Kazuhito Suzuki ${ }^{2}$, Kaichi Nishiwaki ${ }^{2}$ and Hiroshi Yaguchi ${ }^{1}$
}

\begin{abstract}
:
A 48-year-old woman with polyneuropathy, organomegaly, endocrinopathy, M-protein, skin change (POEMS) syndrome suddenly presented with numbness of her right upper limb. Magnetic resonance imaging showed multiple acute infarctions in her left cerebrum, and magnetic resonance angiography (MRA) showed multiple intra-cranial vascular lesions, which contrasted with previously normal MRA results obtained eight months prior to the stroke. After completing successful treatment for POEMS syndrome, there were no recurrent stroke episodes. A six-month follow-up scan showed that although the vascular lesions did not progress, they did not improve much either. POEMS syndrome is associated with the rapid extension of large blood vessels-vasculopathy-resulting in nearly irreversible brain lesions.
\end{abstract}

Key words: POEMS syndrome, ischemic stroke, vasculopathy

(Intern Med 58: 3573-3575, 2019)

(DOI: 10.2169/internalmedicine.3279-19)

\section{Introduction}

polyneuropathy, organomegaly, endocrinopathy, Mprotein, skin change (POEMS) syndrome is a multi-systemic disease associated with plasma cell monoclonal proliferation. It is characterized by and named after the following symptoms: polyneuropathy, organomegaly, endocrinopathy, monoclonal gammopathy and skin changes (1). Although ischemic stroke is not a common feature of POEMS syndrome, it is believed to be a predictor of a poor prognosis $(2,3)$. Indeed, multiple vascular anomalies have been previously reported in patients with POEMS syndrome. However, whether or not a link between the two truly exists is unclear (4-7).

We herein report the case of an ischemic stroke patient with POEMS syndrome who was monitored throughout the clinical course of vasculopathy.

\section{Case Report}

A 48-year-old woman with hypothyroidism was admitted to our hospital with numbness, pitting edema, hyperpigmen- tation, and hirsutism of her lower extremities. She had no history of smoking, alcohol consumption, or recreational drug use.

Eight months prior to admission, she underwent brain magnetic resonance imaging (MRI) and magnetic resonance angiography (MRA) because of mild headaches. Neither modality revealed any specific findings (Figure A). The neurological examination following her admission revealed diffuse muscle weakness in her limbs, a superficial sensation disturbance in her peripheral limbs, and an absence of the deep tendon reflex. Blood tests showed mild hypothyroidism, a platelet count of $256 \times 10^{9} / \mathrm{L}$, prothrombin timeinternational normalized ratio of 1.19 , activated partial thromboplastin time of 27.5 seconds, fibrinogen of $356 \mathrm{mg} /$ $\mathrm{dL}$, fibrin/fibrinogen degradation products of $5 \mu \mathrm{g} / \mathrm{mL}$, and D-dimer of $0.9 \mu \mathrm{g} / \mathrm{mL}$, and electrophoresis revealed elevated $\operatorname{IgA}-\lambda$ paraprotein levels. The vascular endothelial growth factor (VEGF) serum levels were very high at $2,800 \mathrm{pg} / \mathrm{mL}$ (normal 62-707 pg/mL). Cerebrospinal fluid analyses revealed that protein levels were elevated $(214 \mathrm{mg} / \mathrm{dL})$, the cellular and glucose content was normal, and culture specimens were negative. Nerve conduction tests showed symmetrical sensorimotor demyelinating neuropathy. Hepa-

${ }^{1}$ Department of Neurology, Kashiwa Hospital, The Jikei University School of Medicine, Japan and ${ }^{2}$ Department of Clinical Oncology/Hematology, Kashiwa Hospital, The Jikei University School of Medicine, Japan

Received: May 2, 2019; Accepted: June 16, 2019; Advance Publication by J-STAGE: July 31, 2019

Correspondence to Dr. Kenichi Sakuta, kenichisakuta@yahoo.co.jp 

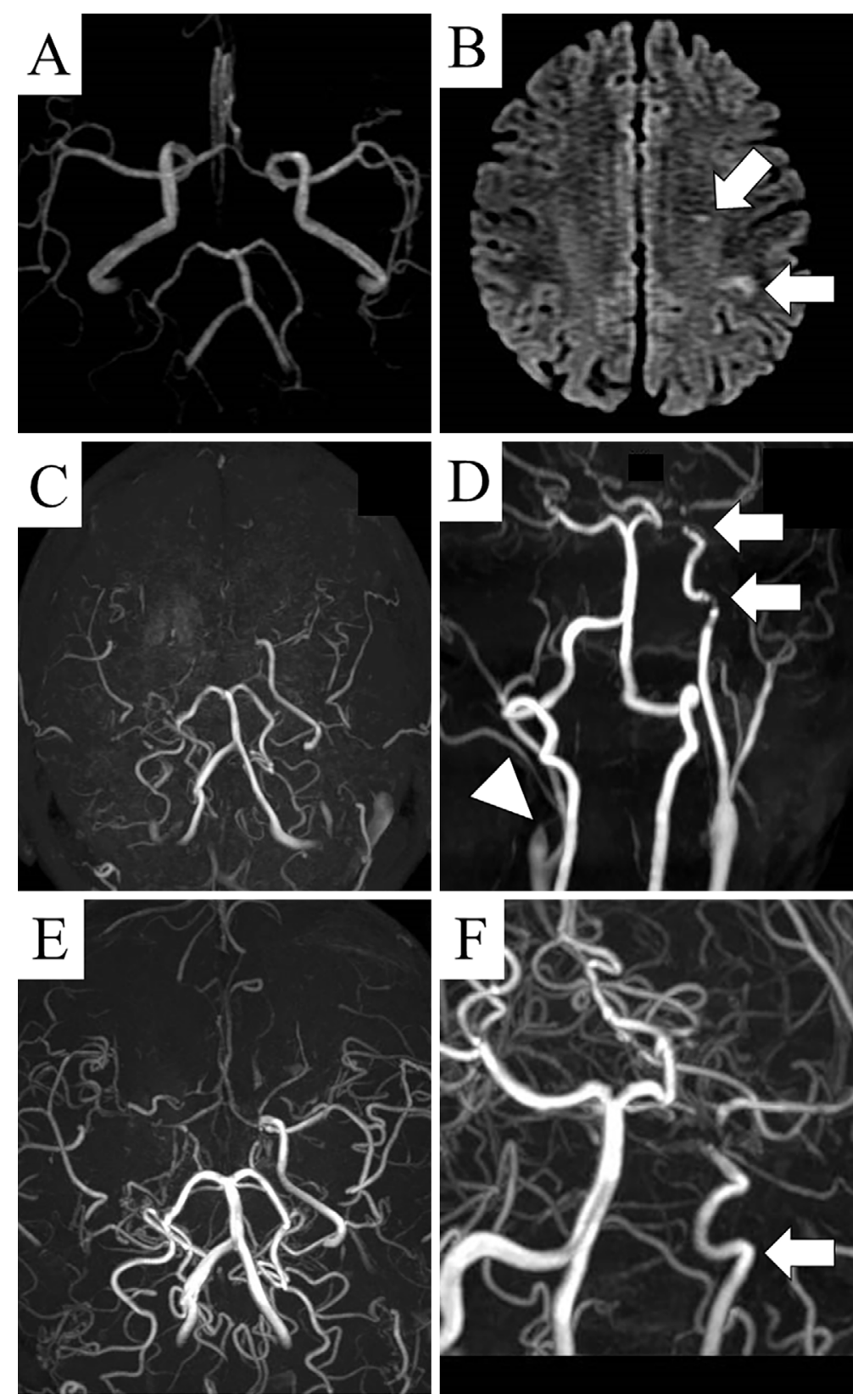

Figure. A: Brain magnetic resonance angiography (MRA) scans taken eight months prior to the first admission. No specific vascular lesions can be seen. B: Diffusion-weighted magnetic resonance imaging (MRI) scans obtained on the day of the stroke onset. Multiple acute ischemic stroke lesions can be seen in the left cerebrum (arrow). C: Brain MRA scans taken on the day of the stroke onset. The right internal carotid artery (ICA), right middle cerebral artery (MCA), and bilateral anterior cerebral artery (ACA) were found to be occluded. D: Brain-carotid MRA scan taken three days after the stroke onset. The right ICA is not depicted from its origin (arrowhead), and a tandem stenotic lesion in left ICA was detected (arrow). The proximal vascular stenosis of the tandem lesion was not detected by brain MRA taken on the day of the stroke onset. E, F: Brain MRA scans taken six months after the stroke onset. The bilateral ACAs, left MCA, and proximal stenosis of the tandem lesion (arrow) showed mild improvement.

tosplenomegaly, pleural effusion, and ascites were detected with chest-abdominal computed tomography. A bone marrow biopsy did not reveal plasma cell myeloma or amyloidosis. The patient was diagnosed with POEMS syndrome, and treatment was initiated with dexamethasone.

Four days later, she suddenly presented with numbness in her right upper limb. We immediately performed brain MRI and MRA, which revealed multiple infarctions in the left cerebrum (Figure B). The right internal carotid artery (ICA), right middle cerebral artery (MCA), and bilateral anterior cerebral artery (ACA) were found to be occluded (Figure C). Carotid MRA performed three days after the onset of stroke showed a tandem stenotic lesion in the left ICA, the proximal one of which could not be detected in the pre- 
vious brain MRA scan (Figure D). Dehydration was not noted based on her laboratory data. Antinuclear antibodies, anti-SS-A/SS-B antibodies, antiphospholipid antibodies, and antineutrophil cytoplasmic antibodies were all negative, ruling out possible rheumatic disease. Carotid ultrasonography revealed circumferential wall thickening in the right ICA and a to-and-fro pattern on color Doppler. Transthoracic echocardiography showed no abnormalities, and an electrocardiogram showed no signs of atrial fibrillation. She did not have any symptoms related to cortical ischemia (hemispatial neglect, conjugate deviation of eyes, or aphasia). The etiology of the ischemic stroke was presumed to be artery to artery embolism.

Dual antiplatelet therapy with aspirin $200 \mathrm{mg} /$ daily and cilostazol $200 \mathrm{mg} /$ daily was prescribed immediately. Within a few days, the right arm numbness dissipated. The patient was initially treated with bortezomib and dexamethasone, followed by a high dose of chemotherapy with melphalan $\left(200 \mathrm{mg} / \mathrm{m}^{2}\right)$ and then autologous hematopoietic stem cell transplantation. After these treatments, her limb weakness and sensory disturbances were found to gradually improve, and her VEGF serum levels decreased to $565 \mathrm{pg} / \mathrm{mL}$. The patient did not experience any ischemic stroke relapses during the next six months. She underwent brain MRA six months after the stroke onset, which showed a little improvement in her lesions (Figure E and F).

\section{Discussion}

The novel finding of this case report is the rapid progression of vascular lesions in a patient with POEMS syndrome. Furthermore, the vascular lesions did not improve much despite the successful treatment of POEMS syndrome.

To our knowledge, this is the first case documenting the initial clinical course of vasculopathy leading to an ischemic stroke in a patient with POEMS syndrome. Cerebral infarctions are not a rare complication in patients with POEMS syndrome, which have been reported in $17 \%$ of cases as an initial symptom with a 5-year risk of $13.4 \%(2,4,8)$. Even though ischemic stroke patients with POEMS syndrome are less likely to have vascular risk factors than general stroke patients, most of them have been reported as having structural vascular anomalies $(2,4,5)$. The findings of the present case report are in agreement with those of previous studies regarding the absence of vascular risk factors and abnormality of coagulation system. Ischemic stroke in the present case arose from vasculopathy, not cardioembolism or lacuna. It is noteworthy that multiple vascular lesions developed parallel to the deterioration of the POEMS symptoms in such a short time frame ( 8 months), suggesting a possible close relationship between POEMS syndrome and vascular lesions. At the very least, we can conclude that the etiology of vasculopathy in the present case was not typical athero- sclerosis. Furthermore, chemotherapy, which can cause vasculopathy, had not been administered prior to the vasculopathy diagnosis.

The present findings indicate that the severity of the POEMS syndrome, which is represented by high levels of inflammatory cytokines, such as VEGF, is correlated with the extent of vascular lesions. Cerebral large-vessel vasculitis is assumed to be the cause of vascular lesions and ischemic strokes in patients with POEMS syndrome $(2,3,7)$. However, the present case showed little changes in the vascular lesions six months later, despite the successful treatment of POEMS syndrome, suggesting that inflammation may not be the only cause of vascular structural changes. Several articles have suggested that non-inflammatory structural changes cause vasculopathy in patients with POEMS syndrome $(4,6)$.

Because the vasculopathy appears to be nearly irreversible, the early initiation of treatment is highly recommended in order to prevent vascular lesions and cerebral infarctions in patients with POEMS syndrome.

\section{The authors state that they have no Conflict of Interest (COI).}

\section{Acknowledgement}

We thank Patrice Voss, Ph.D., for editing a draft of this manuscript.

\section{References}

1. Dispenzieri A. POEMS syndrome: update on diagnosis, riskstratification, and management. Am J Hematol 90: 951-962, 2015.

2. Fu FW, Rao J, Zheng YY, Wang HL, Yang JG, Zheng GQ. Ischemic stroke in patients with POEMS syndrome: a case report and comprehensive analysis of literature. Oncotarget 8: 8940689424, 2017

3. Lee MR, Choi HJ, Lee EB, Baek HJ. POEMS syndrome complicated by extensive arterial thromboses. Clin Rheumatol 26: 19891992, 2007.

4. Dupont SA, Dispenzieri A, Mauermann ML, Rabinstein AA, Brown RD Jr. Cerebral infarction in POEMS syndrome: incidence, risk factors, and imaging characteristics. Neurology 73: 13081312, 2009.

5. Garcia T, Dafer R, Hocker S, Schneck M, Barton K, Biller J. Recurrent strokes in two patients with POEMS syndrome and Castleman's disease. J Stroke Cerebrovasc Dis 16: 278-284, 2007.

6. Mitsutake A, Matsumoto $H$, Hatano $K$, Irie $K$, Tsukada $N$, Hashida $\mathrm{H}$. Lenalidomide-induced ischemic cerebrovascular disease in polyneuropathy, organomegaly, endocrinopathy, monoclonal gammopathy, and skin changes syndrome. J Stroke Cerebrovasc Dis 27: e102-e103, 2018.

7. Forlivesi S, Bacchin R, Cappellari M, Dall'Ora E, Curro Dossi R, Bonetti B. Cerebral large-vessel vasculitis as an unusual manifestation of POEMS syndrome. Neurol Sci 38: 1529-1531, 2017.

8. Wang Z, Xiong L, Zu H. Recurrent stroke as the clinical onset of POEMS syndrome. J Clin Neurol 13: 199-200, 2017.

The Internal Medicine is an Open Access journal distributed under the Creative Commons Attribution-NonCommercial-NoDerivatives 4.0 International License. To view the details of this license, please visit (https://creativecommons.org/licenses/ by-nc-nd/4.0/).

(C) 2019 The Japanese Society of Internal Medicine Intern Med 58: 3573-3575, 2019 